\title{
Comparison of the Calcaneal Pitch Angle and Modified Projection Area Per Length Squared Method for Medial Longitudinal Arch Evaluation of the Foot
}

\author{
Ilgaz Akdoğan', Semih Akkaya², Nuray Akkaya³, Esat Kıter² \\ ${ }^{1}$ Department of Anatomy, Faculty of Medicine, Pamukkale University, Denizli, Turkey \\ ${ }^{2}$ Department of Orthopedics and Traumatology, Faculty of Medicine, Pamukkale University, Denizli, Turkey \\ ${ }^{3}$ Department of Physical Medicine and Rehabilitation, Faculty of Medicine, Pamukkale University, Denizli, Turkey
}

\begin{abstract}
Objective: To compare the calcaneal pitch angle (CPA) values measured on direct lateral radiographs of feet, and the modified projection area per length squared (PAL), which was calculated as a new method for the evaluation of the medial longitudinal arch (MLA) of the foot.

Material and Methods: Direct lateral radiographs of patients who had weightbearing feet radiographies for any reason except trauma were retrospectively obtained from the archives. Direct lateral radiographs of the feet were printed and a transparent sheet was placed on it. A straight line was drawn between the most plantar process of the calcaneus and the head of the first metatarsal bone for the calculation of the PAL of the MLA. Two semilunar arcs were drawn upon this straight line. PAL1 and PAL2 were estimated using a point-counting technique. The CPA, lateral talo-calcaneal angles (LTCA), and talo-first metatarsal angles (TFMA) were measured. The correlations between PAL1, PAL2 of right and left feet and CPA, LTCA, and TFMA were explored.
\end{abstract}

Results: Fifty patients (27 females, 23 males) with a mean age of 40.12 (4-78) years were evaluated. Significant correlations were detected between PAL1, PAL2 and CPA, and TFMA for both right and left feet $(p<0.05)$.

Conclusion: A significant correlation was detected between the modified PAL method as a new technique and the standard CPA method for MLA evaluation. The PAL method is suggested as a simple and practical method for MLA evaluation.

Key Words: Medial longitudinal arch, calcaneal pitch angle, projection area per length squared

Received: 30.01 .2012

Accepted: 15.05 .2012

\section{Introduction}

The longitudinal arch of the foot is composed of medial and lateral parts. Functionally, both parts act as a unit with the transverse arch, spreading the weight in all directions. The medial longitudinal arch (MLA) is higher and more important than the lateral longitudinal arch. The MLA is composed of the calcaneus, talus, navicular, three cuneiforms, and first three metatarsal bones $(1,2)$. MLA has been used as the main reference for diagnosing flatfoot or assessing treatment results. Evaluation of the MLA of the foot is the most important component in the determination of the pes planus and cavus (3). There is no consensus for MLA measurement in Orthopedics; however, there are several suggested techniques, including anthropometric and radiologic measurements, footprints, and photographic analysis (4-7). Radiologic measurements are usually based on the evaluation of different angles. The calcaneal pitch angle (CPA) method is one of these methods. The measurement of the surface area of irregularly shaped structures by a point-counting method was described previ- ously as an efficient approach for obtaining the required cut surface areas of the MLA structure (8).

The purpose of this study is the comparison of the CPA values measured on the direct lateral radiographs of feet and the modified projection area per length squared (PAL), which is a new method (9).

\section{Material and Methods}

Direct lateral radiographs of patients who had weightbearing feet radiographies for any reason except trauma were retrospectively obtained from the archives of Pamukkale University, Faculty of Medicine, Denizli, Turkey. Direct lateral radiographs of the right and left feet were printed and a transparent sheet was placed over them for PAL measurement. A straight line was drawn between the most plantar process of the calcaneus and the head of the first metatarsal bone for the calculation of the PAL of the MLA. Two arc (semilunar) lines were drawn on this straight line. One of the arc lines was tangential to the inferior margin of the calcaneus, 
lowermost calcaneocuboid joint, base of first metatarsal and head of first metatarsal, and the other crossed the sustantaculum tali and lower part of the navicular (Figure 1). Both areas (PAL1 and PAL2) were estimated using a point-counting technique. PAL measurements were performed by the author IA.

The point-counting grid, which has point sets at distinct densities on a transparent sheet, could be used to estimate the surface area of sections or projections (10). For this reason, the testing system was superimposed on the radiographies, and the number of points hitting the objects was used to estimate the total surface area of the projection. A test point is a "+" shaped line and is said to hit the object if the upper right corner of the intersection of the cross lines on the test system lies inside the object. After each superimposition, the number of test points hitting the structure of interest on the projections was counted, and the area of each projection was estimated by multiplying the number of points by the area they represent. The formula for the surface area of the projection can be written as follows:

Projection Area $=\mathrm{a} / \mathrm{p} \times \Sigma \mathrm{P}$, where $\Sigma \mathrm{P}$ denotes the point counts and $(a / p)$ represents the area associated with each test point.

The number of points hitting the semilunar region was recorded. The point-counting method was repeated three times and average values were taken. The projection area of the semilunar region was calculated by multiplying $0.25 \mathrm{~cm}^{2}$ by the number of counted points (Figure 2). The straight line was measured with a ruler. The estimated area was divided by the square of the length of the straight line, and the final data for PAL1 and PAL2 were obtained. A decrease in the PAL measurement means that the arch of the foot decreases. The calculation formula is (9):

Projection area per length squared $(\mathrm{PAL})=\left[(\mathrm{a} / \mathrm{p} \times \Sigma \mathrm{P}) / \mathrm{I}^{2}\right] \times 100$, where $\Sigma \mathrm{P}$ denotes the point counts, $(\mathrm{a} / \mathrm{p})$ represents the area associated with each test point, and $(I)$ is the measured length of the straight line between the most plantar process of the calcaneus and the head of the first metatarsal bone.

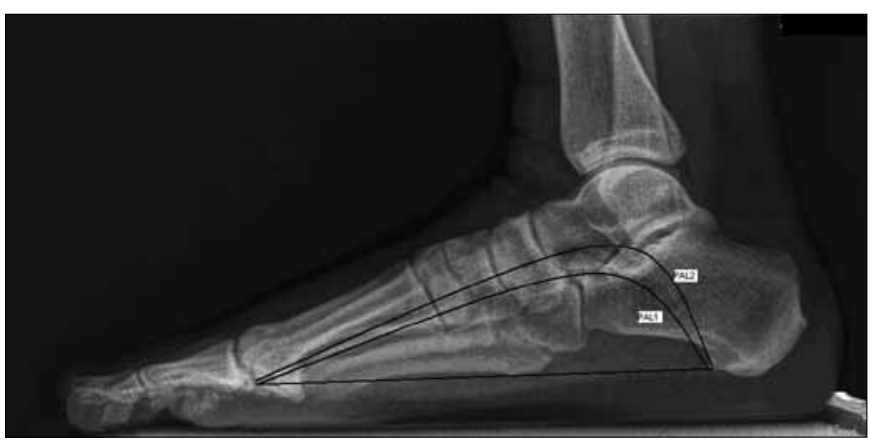

Figure 1. Lateral radiograph of foot showing the area drawn for the estimation of Projection area per length (PAL1 and PAL2)

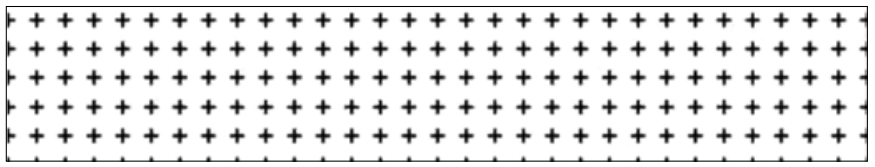

Figure 2. The point-grid
The CPAs, lateral talocalcaneal angle (LTCA), and talofirst metatarsal angle (TFMA) were measured on the same radiographs without a transparent sheet for each subject, as described by Simons and Vanderwilde et al. $(11,12)$. The CPA is an angle that describes the calcaneal inclination that is decreased in flatfoot (Figure 3). The LTCA is used to identify the varus angulation or equinus of the hindfoot, and the TFMA is a measure of the forefoot and hindfoot, which becomes wider in flatfoot. Feet angles were measured by the author SA.

Statistical analysis was performed using the SPSS 17.0 for Windows software package. Descriptive statistics were used, and the PAL and angle measurements were represented as means \pm standard deviations. The correlation between PAL1, PAL2 calculations of right and left feet and CPA, LTCA, and TFMA was determined using the two-tailed Pearson correlation test, and statistical significance was considered to be $\mathrm{p}<0.05$.

\section{Results}

In total, 50 subjects ( 27 females and 23 males) were evaluated (mean age: 40.12 years, min: 4 years, max: 78 years). The mean \pm SD of the right PAL1, PAL2, and CPA were $8.28 \pm 1.41$, $11.26 \pm 11.73$, and $22.98 \pm 4.01$, respectively. The mean \pm SD of the left PAL1, PAL2, and CPA were $8.37 \pm 1.41,11.21 \pm 1.75$, and $21.52 \pm 4.32$, respectively. The mean $\pm S D$ of the right LTCA, left LTCA, right TFMA, and left TFMA were 36.44 \pm 5.21 , $36.86 \pm 5.35$, and $12.6 \pm 1.63,11.7 \pm 2.41$, respectively (Table 1).

A significant correlation was detected between the right PAL1 and right PAL2 and right CPA ( $r=0.644, r=0.595$; $p=0.001, p=0.001)$. Another significant correlation was detected between the left PAL1 and left PAL2 and left CPA $(r=0.703, r=0.769 ; p=0.001, p=0.001)$ (Table 2).

There were no significant correlations between PAL1, PAL2, and LTCA for both right and left feet.

A significant correlation was detected between the right PAL1, right PAL2, and right TFMA ( $r=0.365, r=0.409 ; p=0.009$, $p=0,003)$. Another significant correlation was detected between the left PAL1, left PAL2, and left TFMA ( $r=0.353$, $r=0.434 ; p=0.012, p=0.002$ ) (Table 2).

Table 1. Measurements of Projection area per length squared, and foot angles

\begin{tabular}{|lll|}
\hline & $\begin{array}{l}\text { Right side } \\
\text { Mean } \pm \text { standard } \\
\text { deviation }\end{array}$ & $\begin{array}{l}\text { Left side } \\
\text { Mean } \pm \text { standard } \\
\text { deviation }\end{array}$ \\
\hline PAL1 & $8.28 \pm 1.41$ & $8.37 \pm 1.41$ \\
PAL2 & $11.26 \pm 11.73$ & $11.21 \pm 1.75$ \\
Calcaneal pitch angle & $22.98 \pm 4.01$ & $21.52 \pm 4.32$ \\
Talocalcaneal angle & $36.44 \pm 5.21$ & $36.86 \pm 5.35$ \\
Talo-first metatarsal & $12.6 \pm 1.63$ & $11.7 \pm 2.41$ \\
angle & & \\
\hline PAL: Projection area per length squared & \\
\hline
\end{tabular}




\section{Discussion}

Medial longitudinal arch measurement is an important component of the evaluation of flatfoot. Angle and height measurements of the MLA are determined from lateral weightbearing roentgenograms of the feet. According to the results of the present study, since significant correlation was detected between the PAL and CPA, the PAL method can be an alternative technique for MLA radiographic evaluation.

Surface area measurement of irregularly shaped structures by a point-counting method was described as an efficient approach for obtaining the required cut surface areas of the structure (8). The PAL method was previously suggested as an alternative to the Cobb method for the radiographic evaluation of the degree of lumbar lordosis when ImageJ's estimation was accepted as the gold standard (9). Authors stated that the Cobb method caused variability in the measurements because of vertebral end plate architecture variability, and offered that the PAL method may be a useful technique for the evaluation of the surface area of the semilunar region for lumbar lordosis (9). They argued that, because of the good agreement between planimetry and point-counting methods, the PAL method could be applied without requiring any software. The current study indicated that the PAL method can be used as an alternative method for measuring the semilunar irregular shape of the MLA in weight bearing lateral roentgenograms of the foot.

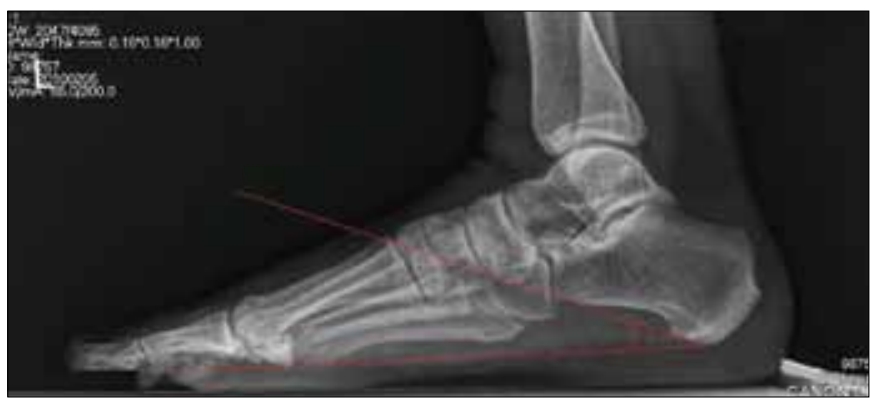

Figure 3. Lateral radiograph of foot showing the CPA

Table 2. Relationships between measurements of Projection area per length squared and Calcaneal pitch Angles, Talo-first metatarsal angles

\begin{tabular}{|lll|}
\hline & r & p \\
\hline Right PAL1-Right Calcaneal pitch Angle & 0.644 & 0.001 \\
Right PAL2-Right Calcaneal pitch Angle & 0.595 & 0.001 \\
Left PAL1-Left Calcaneal pitch Angle & 0.703 & 0.001 \\
Left PAL2-Left Calcaneal pitch Angle & 0.769 & 0.001 \\
Right PAL1-Right Talo-first metatarsal angle & 0.365 & 0.009 \\
Right PAL2-Right Talo-first metatarsal angle & 0.409 & 0.003 \\
Left PAL1-Left Talo-first metatarsal angle & 0.353 & 0.012 \\
Left PAL2-Left Talo-first metatarsal angle & 0.434 & 0.002 \\
\hline PAL: Projection area per length squared & & \\
\hline
\end{tabular}

Although there are many studies comparing the radiological measures for diagnosing flatfoot $(8,13-15)$, there is no consensus on the radiologic evaluation of the MLA. While Younger et al. (14) reported that the TFMA was an accurate radiographic identifier, Sensiba et al. (15) suggested that CPA had a higher interobserver reliability. The relationships of the PAL method with CPA, LTCA, and TFMA were examined in the present study. According to our results, although the LTCA was correlated with the CPA, there was no correlation between the LTCA and the PAL method.

There are many methods other than radiographic measurements for evaluating the MLA structure (16-18). A study examining the correlation of the measurements from footprints and radiographies for flatfoot evaluation contains one of these methods (16). The authors suggested that measurement of the subarch angle significantly correlated with radiographic measurements, and it was easily obtained from a capacitive forceplate (16). In another study, significant correlations were reported between Harris mat imprints and radiographic foot angles, and use of the Harris mat imprint was suggested as an effective method for quantifying flatfoot (17). Wearing et al. (18) reported that digitized videofluoroscopy of calcaneal sagittal plane motion was more beneficial for the assessment of arch function during gait, and authors suggested that calcaneal pitch may result in errors of foot function during gait. They suggested that the CPA may be used as an indicator of a rare foot position only (18). Although there are many benefits of these reported methods for evaluating the MLA, they are time consuming and require expensive, special equipment or software In contrast, the PAL method in our study has high correlation with the CPA, and is an economic and practical technique for MLA structure evaluation without special software or equipment.

Sensiba et al. (15) reported that the CPA had the highest interobserver reliability compared to other radiologic measurements for the radiologic quantification of adult-acquired flatfoot deformity, which is a difficult condition to quantify radiographically. CPA is the preferred method for the determination of the pes planus and cavus in clinical practice. In our study, we searched for relationships of the PAL method with CPA, and TFMA, and found that the PAL method had a high correlation with the CPA, and moderate correlation with the TFMA for the evaluation of the MLA. An increase in the PAL will result from an increase in the pes cavus. In the present study, the cut-off points of PAL for normal and pathologic measurements for medial longitudinal arch were not described. Therefore, a variety of measurements for the assessment of the cut-off points are necessary.

\section{Conclusion}

A significant correlation was detected between the modified PAL method as a new technique and the classical CPA method, and the TFMA method for MLA evaluation. The PAL method is suggested as a simple, objective, and practical method for the evaluation of MLA. This method is not only inexpensive and easy, but it can also be an alternative for measuring the MLA on routine weight bearing lateral radiographies of feet without having to change the normal procedure. 


\section{Conflict of Interest}

No conflict of interest was declared by the authors.

\section{References}

1. Moore KL, Dalley AF. Clinically Oriented Anatomy, Lippincot Williams and Wilkins, Canada, Fourth edition, 1999.p.640.

2. Standring $S$ (Editor). Gray's Anatomy, The Anatomical Basis of Clinical Practice, Section editor; Vishy Mahadevan, Section: Pelvic girdle and lower limb, Churchill Livingstone Elsevier, China, Fortieth edition, 2008.p.1450.

3. Kanatlı U, Yetkin H, Cila E. Footprint and radiographic analysis of the feet. J Pediatr Orthop 2001;21:225-8. [CrossRef]

4. Staheli LT, Chew DE, Corbett M. The longitudinal arch. A survey of eight hundred and eighty-two feet in normal children and adults. J Bone Joint Surg Am 1987;69:426-8.

5. Viladot A. Surgical treatment of the child's flatfoot. Clin Orthop Relat Res 1992;283:34-8.

6. Volpon JB. Footprint analysis during the growth period. J Pediatr Orthop 1994;14:83-5. [CrossRef]

7. Saltzman CL, Nawoczenski DA, Talbot KD. Measurement of the medial longitudinal arch. Arch Phys Med Rehabil 1995;76:45-9. [CrossRef]

8. Yalçin N, Esen E, Kanatli U, Yetkin H. Evaluation of the medial longitudinal arch: a comparison between the dynamic plantar pressure measurement system and radiographic analysis. Acta Orthop Traumatol Turc 2010;44:241-5. [CrossRef]
9. Kuru O, Sahin B, Kaplan S. Alternative approach to evaluating lumbar lordosis on direct roentgenograms: projection area per length squared. Anat Sci Int 2008;83:83-8. [CrossRef]

10. Sahin B, Emirzeoglu M, Uzun A, Incesu L, Bek Y, Bilgic $S$, et al. Unbiased estimation of the liver volume by the Cavalieri principle using magnetic resonance images. Eur J Radiol 2003;47:164-70. [CrossRef]

11. Simons GW. A standardized method for the radiographic evaluation of clubfeet. Clin Orthop Relat Res 1978;135:107-18.

12. Vanderwilde R, Staheli LT, Chew DE, Malagon V. Measurements on radiographs of the foot in normal infants and children. $J$ Bone Joint Surg Am 1988;70:407-15.

13. Lo HC, Chu WC, Wu WK, Hsieh H, Chou CP, Sun SE, et al. Comparison of radiological measures for diagnosing flatfoot. Acta Radiol 2012;53:192-6. [CrossRef]

14. Younger AS, Sawatzky B, Dryden P. Radiographic assessment of adult flatfoot. Foot Ankle Int 2005;26:820-5.

15. Sensiba PR, Coffey MJ, Williams NE, Mariscalco M, Laughlin RT. Inter-and intraobserver reliability in the radiographic evaluation of adult flatfoot deformity. Foot Ankle Int 2010;31:141-5. [CrossRef]

16. Chen $\mathrm{CH}$, Huang $\mathrm{MH}$, Chen TW, Weng MC, Lee $\mathrm{CL}$, Wang GJ. The correlation between selected measurements from footprint and radiograph of flatfoot. Arch Phys Med Rehabil 2006;87:235-40. [CrossRef]

17. Coughlin MJ, Kaz A. Correlation of Harris mats, physical exam, pictures, and radiographic measurements in adult flatfoot deformity. Foot Ankle Int 2009;30:604-12. [CrossRef]

18. Wearing SC, Urry S, Perlman PR, Dubois P, Smeathers JE. Serial measurement of calcaneal pitch during midstance. J Am Podiatr Med Assoc 1999;89:188-93. 